\title{
A Novel Packet Scheduling for Wireless Channels with Adaptive Burst Profile
}

\author{
Do Hoai Nam, Luong Dinh Dung \\ Department of Telecommunications \\ Budapest University of Technology and Economics \\ H-1117, Magyar tudósok körútja 2, Budapest, Hungary \\ Email: \{nam,luong\}@hit.bme.hu
}

\begin{abstract}
This paper proposes an Adaptive Profile Packet Scheduling (APS) algorithm for the down-link of IEEE 802.16 W-MAN, which is identified as a candidate standard for High Attitude Platforms (HAP) in the EU IST CAPANINA project. The proposed APS takes into account different wireless channel conditions observed by different subscriber stations to enhance the whole system utility and preserves the property of the long-term fairness.
\end{abstract}

Keyworlds: Packet Scheduling, Wireless Access, Burst Profiles.

\section{INTRODUCTION}

CAPANINA (Communications from Aerial Platform Networks delivering Broadband Communications for all) is an international cooperation supported by the European Union under Framework 6 Research Programme. The CAPANINA project will develop wireless and optical broadband technologies for use on High Altitude Platforms (HAPs).

IEEE 802.16 is identified as a candidate standard for HAP in the CAPANINA project. The standard specifies QoS classes for the connection on the uplink. However, the scheduling algorithms to realize QoS requirements on the uplink and scheduling algorithms on the downlink are not standardized. The subscriber stations measure the level of the received signal and request the best-suited burst profile from the base station which includes the byte per symbol rate with that the base station can send data to the Subscriber Station (SS) on the downlink. Similarly, the base station measures the level of the received signal from subscriber stations and decides upon the best burst profile for each of them.
Consequently the selected burst profile is used for the respective subscriber station on the uplink.

Typically a HAP is an airship that floats at an altitude of around $20 \mathrm{~km}$, well above any normal aircraft but being in the stratosphere, substantially below orbiting satellites. CAPANINA will deliver low cost broadband communications services to small office and home users at data rates up to $120 \mathrm{Mbit} / \mathrm{s}$ - a staggering 2000 faster than today's dial-up modems and more than 200 times faster than a typical "wired" broadband facility [1].

In such environment, effective packet scheduling algorithms should consider channel conditions in order to enhance system performance. It has been shown that while General Processor Sharing (GPS) and Packet Fair Queuing (PFQ) algorithms guarantees fairness and guaranteed service in wired networks, they cannot satisfy both in a wireless environment where wireless channels may be blocked by errors. Eugene et al.[7] define a set of desirable properties called CIF that a scheduling algorithm should satisfy in the context of location-dependent channel errors. They also propose a scheduling algorithm called CIF-Q and prove that CIF$\mathrm{Q}$ achieve all CIF properties.

In many works, e.g. [6], [7], the wireless channel is modeled with two states: error-free and erroneous. Sessions in error state will offer their service share to sessions in the error-free state and claim it back when they switch to the error-free state. Recently, different adaptive mechanisms such as adaptive modulation and channel coding have been proposed to enhance system 
capacity. The channel can be in multiple states and each state is assigned with a combination of mechanisms called burst profile to maximize the data rate while maintain the bit error rate (BER) under a certain threshold.

To use the burst profiles in an efficient manner, packet scheduling needs to be developed to achieve high system throughput while maintaining QoS and fairness conditions. On the downlink, since a minimum reserved rate is the basic QoS parameter negotiated by a connection within an 802.16 scheduling service, the class of latency-rate scheduling algorithms is particularly suited for implementing the schedulers in the 802.16 MAC as discussed in [9]. Some other works on the packet scheduling on the up-link [4], [5], [8] of IEEE 802.16 focused on satisfying requirements of different QoS classes but they did not consider the dynamics of the wireless channel's condition.

In our previous work [10], the cross-layer packet scheduling algorithm was proposed in multi-state downlink wireless channel of IEEE 802.16 W-MAN networks [2] operating in a point-to-multi-point communication scenario, which takes into account different wireless channel conditions observed by subscriber stations to enhance the system throughput. The additional aim is to preserve the property of the long-term fairness and the guaranteed rate for each user, as well as simple implementation and low complexity. The efficiency of scheduling algorithm depends on the freedom degree $p$, which controls fairness for all users, but $p$ factor is vary on different systems and difficult to obtain.

In this work, we solve the $p$ freedom degree problem with a novel approach. We propose a scheduling algorithm which have a low complexity and is easy to be implemented. The algorithm is based a cross-layer system design exchange information between the MAC and physical layers using Adaptive Modulation and Coding (AMC) technique. Our scheduling algorithm is referred to as the Adaptive Profile Scheduling (APS), which can guarantee minimum rate and long-term fairness while maintaining the high system utility.
Moreover, this work does not heavily depend on the specific properties of IEEE 802.16 and therefore, the algorithms can migrate to other wireless access environments, which apply adaptive profiles.

The rest of the paper is organized as follows. In Section II, we briefly describe the operation scenario. In Section III, we present the proposed scheduling algorithm. In Section IV, we investigate and compare the performance of the APS with the Fair Queuing. Section V concludes our work.

\section{OPERATION SCENARIO}

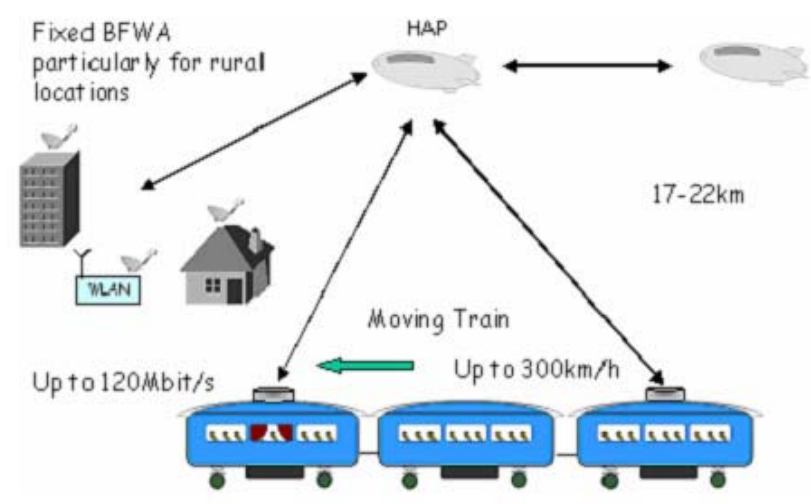

Fig 1. A typical operation scenario in HAP systems

Consider a wireless network with a base station (BS) and $N$ subscriber stations (SS) working in point-tomulti-point communication scenario. As shown in Fig. 1 , in a typical operation scenario of HAP systems targeted in CAPANINA project, both fixed and mobile SSs are considered with the mobile SS's velocity up to $400 \mathrm{~km} / \mathrm{s}$. The data packets are segmented into fixed size ARQ blocks and scheduled on the downlink with Time Division Multiplexing (TDM) and Adaptive Burst Profiles [2]. Data to be sent to SSs are multiplexed in TDM frames which size is $S$ symbols.

An SS measures the received signal level sent by the BS and decides which is the best burst profile for its downlink transmission. The burst profile change request then can be sent to the BS on the up-link. Let BP is the set of pre-defined burst profiles. At any point of time, each SS is assigned with a burst profile which consists of a modulation and a channel coding technique, i.e. for 
the SS $i$ at time frame $k$, a burst profile $b p(i, k)$ is chosen from BP with that a symbol can carry $b p s(b p(i, k))$ bytes of data. We assume that the chosen burst profile is efficient enough to transfer data successfully to the SS.

At each frame, e.g. at frame $k$, the $\mathrm{BS}$ with a given set of SSs and their assigned burst profiles must decide how to transfer data. That is, $n(i, k)$ symbols are assigned to SS $i$ to carry its data, where $\sum_{i=1}^{N} n(i, k) \leq S$

The data amount of all SS $i$ packet in frame $k$ :

$$
D(i, k)=n(i, k) b p s(b p(i, k))
$$

The data amount of all SS-s packed in frame k can be calculated as

$$
D(k)=\sum_{i=1}^{N} D(i, k)=\sum_{i=1}^{N} n(i, k) \operatorname{bps}(b p(i, k))
$$

which has an upper bound:

$$
\begin{gathered}
D_{\max }(k)=\max (D(k))=b p s\left(b p_{\text {best }}(k)\right) S, \\
b p_{\text {best }}(k) \in\{b p(i, k)\} \forall i, \\
b p s\left(b p_{\text {best }}(k)\right) \geq b p s(b p(l, k)) \quad \forall l .
\end{gathered}
$$

Therefore, the average amount of data per frame taken over $m$ frame is

$$
D_{\text {avg }}(i, m)=\frac{\sum_{j=1}^{m} D(i, j)}{m},
$$

for SS $i$ and

$$
D_{\text {avg }}(m)=\frac{\sum_{k=1}^{m} D(k)}{m},
$$

for all SSs.

The throughput fairness index is defined according to Jain et al. [13]

$$
I(m)=\frac{\left(\sum_{i=1}^{N} D_{a v g}(i, m)\right)^{2}}{N \sum_{i=1}^{N} D_{a v g}(i, m)^{2}}
$$

The throughput fairness index takes values between 0 and 1. The closer the index to 1 , the fairer the scheduling.

\section{THE ADAPTIVE PROFILE SCHEDULING (APS)}

The objective of the scheduling is to:

- enhance the overall throughput $G$,

- guarantee the long-term fairness,

- provide a throughput guarantee of $r_{i}$ for each SS $i$.

The proposed APS performs as illustrated in Algorithm 1. We assume that each SS will be assigned with a FIFO buffer at the BS. The MAC packets to be sent to SSs are classified according to their receiver SS address and put into the corresponding FIFO buffers.

The main principle of APS is that the SS with better burst profile (higher bps) should borrow resources from the SS with worst burst profile and gives back the borrowed resources when its channel condition is bad. This main principle is combined with some mechanisms to guarantee the minimum rates and fairness. Two token buckets are used: the first token bucket is for the minimum rate guarantee and the second one is for the fairness guarantee purpose.

Denote the FIFO buffer assigned to SS $i$ for storing MAC packets by queue ${ }_{i}$. The actual sizes of the first and second token bucket are $t_{i 1}$ and $t_{i 2}$, respectively. The depth of the token buckets is upper bounded $\left(\max _{1}\right.$ and $\left.\max _{2}\right) \cdot r_{i}$ is the minimum rate guarantee for SS $i$. Slots of each TDM time frame are distributed among SSs after five steps:

(i) In the first step, adequate slots are reserved according to the guaranteed minimum rate of each SS using the first token bucket.

(ii) The second step guarantees the long-term fairness for each SS by limiting the borrowed slots.

(iii) The third step enhances the system throughput by giving higher privilege to SSs with better burst profile.

(iv) In the fourth and fifth steps, the token buckets are refilled.

In the fourth \& fifth steps, the token buckets are 
refilled. The first bucket is refilled once in a rough, but the second can be refilled more than once as the following step of the second and the third steps. Those three steps can be repeated as much as possible in order to solve the starve problem: in some cases, some users have data but they haven't got enough tokens in the second bucket to send out. Some others haven't data, but they have enough tokens to send. Those unused tokens can be redistributed: users can borrow tokens from others to send out their data, and will pay later in another redistribution rough. By this way, we can maintain the long-term fairness of the system.

The detailed code of APS is shown in Algorithm 1.

$$
\begin{aligned}
& \text { Algorithm } 1 \text { Description of the APS } \\
& \text { \{step } 1 \text { - For minimum rate guarantee }\} \\
& \text { filledsymno }=0 \\
& \text { for } i=1 \text { to } N \text { do } \\
& \text { reserve } \\
& s_{i}=\min \left\{\left[\frac{\left\lfloor t_{i 1}\right\rfloor}{b p s(b p(i, k))}\right],\left\lceil\frac{\operatorname{data}_{i}}{b p s(b p(i, k))}\right]\right\} \text { for SS i } \\
& \text { filledsymno }+=s_{i} \text {; } \\
& t_{i 1}-=\min \left\{\text { data }_{i}, t_{i 1}, s_{i} * \operatorname{bps}(b p(i, k))\right\} \\
& \text { data }_{i}-=\min \left\{\text { data }_{i}, s_{i} * \operatorname{bps}(b p(i, k))\right\} \\
& \text { end for } \\
& \text { if filledsymno }=S \text { then } \\
& \text { goto step } 5 \\
& \text { end if } \\
& \text { sharedsymno }=0 \\
& \text { for } i=1 \text { to } N \text { do } \\
& \text { if } t_{i 2}>\max _{2} \text { and } \operatorname{data}_{i}>0 \text { then } \\
& \text { reserve } \\
& s_{i}=\min \left\{\left\lceil\frac{\text { data }_{i}}{b p s(b p(i, k))}\right],\left\lfloor t_{i 2}\right\rfloor-\max _{2}, S-\text { filledsymno }\right\} \\
& \text { for } \mathrm{SS} i \\
& \text { sharedsymno }+=t_{i 2}-\max _{2} \\
& \text { filledsymno }+=s_{i} \\
& \text { data }_{i}-=\min \left\{\text { data }_{i}, s_{i}{ }^{*} \operatorname{bps}(b p(i, k))\right\} \\
& t_{i 2}=\max _{2}
\end{aligned}
$$

if filledsymno $=S$ then end the for loop and goto step 4 end if

end for

end if

\{step 3 - Enhance system throughput\}

$\left\{l_{1}, l_{2}, \ldots, l_{N}\right\}$ is a permutation of $\{1,2, \ldots, N\}$, where:

$\operatorname{bps}\left(b p\left(l_{1}, k\right)\right) \geq \operatorname{bps}\left(b p\left(l_{2}, k\right)\right) \geq \ldots \geq \operatorname{bps}\left(b p\left(l_{N}, k\right)\right)$

for $i=l_{1}$ to $l_{N}$ do

reserve

$s_{i}=\min \left\{\left[\frac{\text { data }_{i}}{\operatorname{bps}(b p(i, k))}\right],\left\lfloor t_{i 2}\right\rfloor, S-\right.$ filledsymno $\}$

for SS $i$

sharedsymno $+=s_{i}$

filledsymno $+=s_{i}$

data $_{i}-=\min \left\{\operatorname{data}_{i}, s_{i} * \operatorname{bps}(\operatorname{bp}(i, k))\right\}$

$t_{i 2}-=s_{i}$

if filledsymno $=S$ then

end if end the for loop and goto step 4

end for

\{step 4 - Refill the second token bucket\}

for $\quad \mathrm{i}=1$ to $\mathrm{N}$ do

$$
t_{i 2}+=\frac{\text { sharedsymno }}{N}
$$

end for

if filledsymno $<S$ then

$$
\text { if } \quad \exists i,\left\{1 \leq i \leq N \mid \text { data }_{i}>0 \text { and } t_{i 2}<1.0\right\}
$$

then

$$
\text { end if goto step } 2
$$

end if

\{step 5 - Refill the first token bucket\}

for $\mathrm{i}=1$ to $\mathrm{N}$ do

$$
t_{i 1}=\min \left\{\max _{1}, t_{i 1}+\frac{r_{i} * 1000 * \text { frametime }}{8}\right\}
$$

end for 


\section{NUMERICAL RESULTS}

In this section, we present some simulation results to illustrate the utility improvement and the fairness of the APS. The algorithm was implemented in the ns-2 network simulator [3] with parameters shown in Table 2. The wireless channel quality is characterized by the received signal-to-noise ratio (SNR) which is partitioned into 6 intervals with the thresholds of $A_{0}, A_{1}, \ldots, A_{6}$ (Table 1), as suggested by Li et al. [12]. Six burst profiles are assigned to these intervals with different bps parameters (Table 3). The finite state Markov channel model was constructed from the Rayleigh fading channel as proposed in [11]. Time variation of the signal levels is characterized by Doppler frequency effect caused by the motion of subscriber stations.

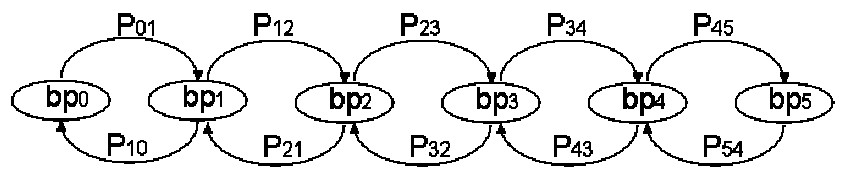

Fig 2. The state transition between the Markov chain's states

The state transition of the Markov chain happens in the frame time basis with the normalized transition probabilities $p_{i j}$ shown in Table IV. In other words, $p_{i j}$ is the probability that the channel state changes to burst profile $j$ given the previous state is $i$ and the Doppler frequency is $10 \mathrm{~Hz}$. Since the time frame of 1 ms is small enough, we can consider $p_{i j}=0$ if $|i-j|>1$.

A transition probability equals to the corresponding normalized probability multiplying the Doppler frequency.

The network shown in Fig. 3 has been used to investigate the performance of APS. The topology consists of 20 FTP client-server pairs, a router and a BS. The servers are connected to the router with a 2 $\mathrm{GB} / \mathrm{s}$ link having a latency of $10 \mathrm{~ms}$. The link between the router and the BS has a bandwidth of $2 \mathrm{~GB} / \mathrm{s}$ and a latency of $50 \mathrm{~ms}$. The BS offers a downlink of 2 $\mathrm{Mbaud} / \mathrm{s}$ for the SSs with the FTP clients. The up-link is emulated by a wired link of $2 \mathrm{~GB} / \mathrm{s}$. In this configuration the wireless link is the bottleneck thus congestion occurs only at the BS. The TDM frame size was chosen to be $2000 \mathrm{symbols} / \mathrm{ms}$. For traffic simulation, an FTP download session has been initiated on each client-server pair. Three different guaranteed minimal bandwidth values: $400 \mathrm{kB} / \mathrm{s}, 600 \mathrm{kB} / \mathrm{s}$ and 800 $\mathrm{kB} / \mathrm{s}$ have been used. During a simulation run, the guaranteed bandwidth has been the same for all users.

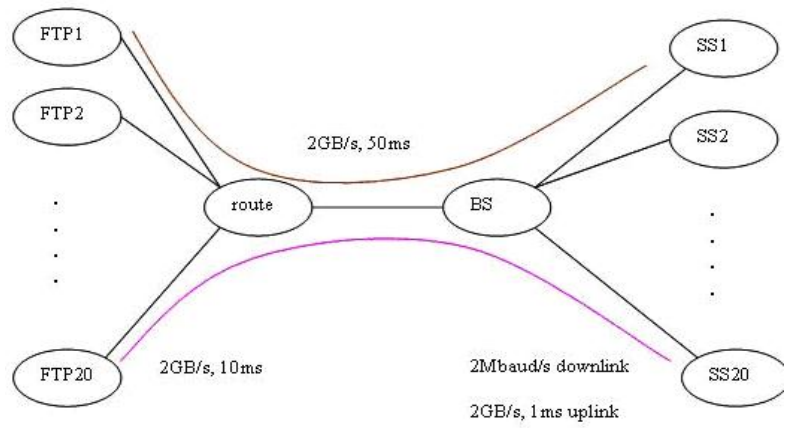

Fig. 3. The simulation topology

\begin{tabular}{|c|c|}
\hline SNR threshold & Value $(\mathrm{dB})$ \\
\hline$A_{0}$ & 0 \\
\hline$A_{1}$ & 6.1 \\
\hline$A_{2}$ & 8.8 \\
\hline$A_{3}$ & 11.6 \\
\hline$A_{4}$ & 16.7 \\
\hline$A_{5}$ & 18 \\
\hline$A_{6}$ & $\infty$ \\
\hline
\end{tabular}

Table 1 - SNR thresholds

The Round Robin algorithm has been used to compare with APS since the Round Robin is the only general algorithm to be used in a multi-state channel environment. We have measured the fairness index of the flows and the average throughput provided by the two algorithms in every case.

\begin{tabular}{|l|c|}
\hline Name & Value \\
\hline TDM frame size $(\mathrm{S})$ & 2000 symbol \\
\hline Channel capacity & $2 \mathrm{Mbaud} / \mathrm{s}$ \\
\hline Number of users(N) & 20 \\
\hline Average SNR $(\rho)$ & $12 \mathrm{~dB}$ \\
\hline Doppler frequency $f_{m}$ & $10 \mathrm{~Hz}$ \\
\hline
\end{tabular}

Table 2 - Simulation parameters 


\begin{tabular}{|c|c|c|}
\hline Burst Profile Id & Intervals & $\begin{array}{c}\text { Bytes per } \\
\text { Symbol (bps) }\end{array}$ \\
\hline bp0 & $\left(A_{0}, A_{1}\right]$ & 24 \\
\hline bp1 & $\left(A_{1}, A_{2}\right]$ & 36 \\
\hline bp2 & $\left(A_{2}, A_{3}\right]$ & 48 \\
\hline bp3 & $\left(A_{3}, A_{4}\right]$ & 72 \\
\hline bp4 & $\left(A_{4}, A_{5}\right]$ & 96 \\
\hline bp5 & $\left(A_{5}, A_{6}\right)$ & 108 \\
\hline
\end{tabular}

Table 3 -Pparameters of burst profiles

As shown in Fig. 4, the improvement of the average throughput can be as high as $23.14 \%$ with $400 \mathrm{kB} / \mathrm{s}$ guaranteed bandwidth values. With different guaranteed bandwidth, the improvement can be $22.17 \%$ for the $600 \mathrm{kB} / \mathrm{s}$ case and $20.66 \%$ for the $800 \mathrm{kB} / \mathrm{s}$ case.

\begin{tabular}{|c|c|c|c|}
\hline Prob. & Value & Prob. & Value \\
\hline $\mathrm{p} 01$ & 0.0027 & $\mathrm{p} 10$ & 0.0089 \\
\hline $\mathrm{p} 12$ & 0.0085 & $\mathrm{p} 21$ & 0.0103 \\
\hline $\mathrm{p} 23$ & 0.0094 & $\mathrm{p} 32$ & 0.0071 \\
\hline $\mathrm{p} 34$ & 0.0056 & $\mathrm{p} 43$ & 0.0047 \\
\hline $\mathrm{p} 45$ & 0.0044 & $\mathrm{p} 54$ & 0.0075 \\
\hline
\end{tabular}

Table 4 - Transition probabilities of the markov chain

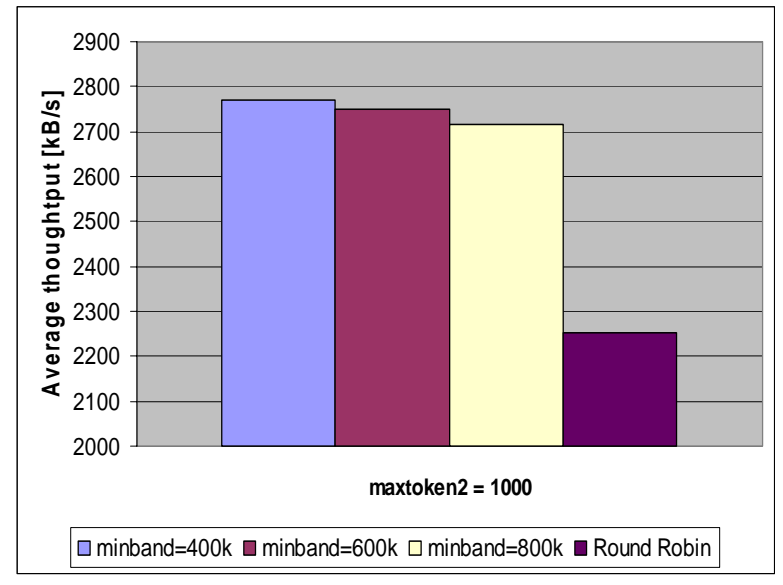

Fig 4. The average throughput of APS and Round Robin versus the minimal guaranteed bandwidth

The simulation results show APS shares symbols fairly among the SSs. The smaller the guaranteed bandwidth the higher the number of unassigned slots. APS shares these unassigned slots effectively since users with good burst profiles receives more slots than users with a bad profile does. Thus, the higher the number of unassigned slots the higher the throughput of the system.

The simulation results show that the long-term fairness is independent of the different scheduling parameters. In all the cases, the long-term fairness index has converged to the value of 1 as shown on Fig. 5 . Thus all user experiencing the same error distribution receives the same throughput for their downloads. If the error model is different for the users then users with a good average SNR may have a higher throughput than users with a bad average SNR. It can be also observed that the minimum bandwidths $r_{i}$ are always guaranteed.

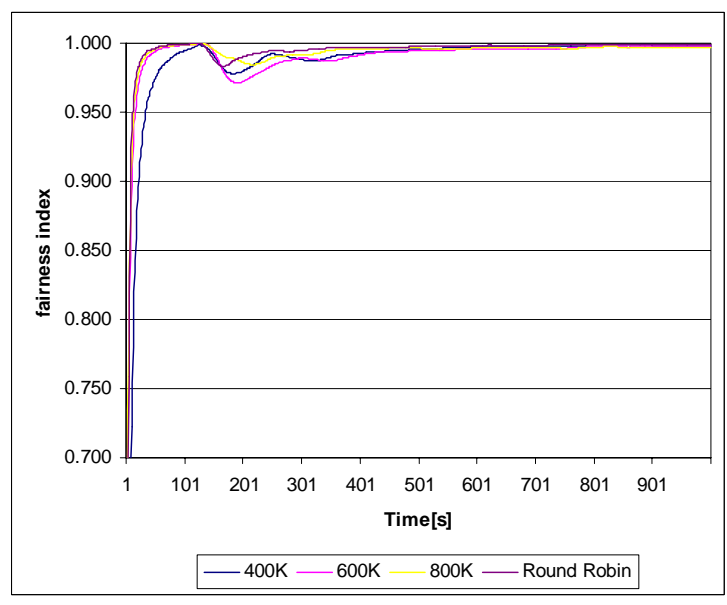

Fig. 5. The fairness index versus time

\section{CONCLUSIONS}

This work has proposed a packet scheduling algorithm called the APS to be used in the down-link of IEEE 802.16 W-MAN networks operating in point-tomulti-point scenarios. Utilizing the different channel conditions seen by subscriber stations, the APS improves the system performance by up to $21 \%$ in a normal configuration parameter setting while preserving the long-term fairness and guarantees minimum rates. The future work will be the extension of the APS from IEEE 802.16 networks into a more general wireless system. Furthermore, the application of analytical models in [14] for the performance evaluation is being considered. 


\section{ACKNOWLEDGMENTS}

The work is performed with the support of the IST CAPANINA project $^{1}$ of the EU Framework 6 programme.

\section{REFERENCES}

[1] CAPANINA project, http://www.capanina.org.

[2] IEEE Std 802.16-2001, IEEE Standard for Local and Metropolitan Area Networks - Part 16: Air Interface for Fixed Broadband Wireless Access Systems.

[3] The Network Simulator - ns-2. http://www.isi.edu/nsnam/ns/.

[4] Mohamed Hawa and David W. Petr. Quality of Service Scheduling in Cable and Broadband Wireless Access Systems. In Proceedings of the Tenth International Workshop on Quality of Service (IWQoS 2002), pp. 247 255, 15-17 May 2002.

[5] GuoSong Chu, Deng Wang and Shunliang Mei. A QoS Architecture for the MAC Protocol of IEEE 802.16 BWA System. In Proceedings of the IEEE 2002 International Conference on Communications, Circuits and Systems and West Sino Expositions, pp: 435 - 439, vol. 1, 29 June 2002.

[6] W. K. Wong, H. Tang, Guo Shanzeng and V. C. M. Leung. Scheduling Algorithm in a Point-to-Multipoint Broadband Wireless Access Network. In Proceedings of VTC 2003-Fall - The IEEE Vehicular Technology Conference, Volume: 3, pp. 1593 - 1597, October 2003.

[7] T. S. Eugene Ng, Ion Stoica, Hui Zhang. Packet Fair Queueing Algorithms for Wireless Networks with Location-Dependent Errors. In Proc. of IEEE INFOCOM 1998 - The Conference on Computer Communications, no. 1, pp. 1103-1111, April 1998.

[8] Yaxin Cao and Victor O. K. Li. Scheduling Algorithms in BroadBand Wireless Networks. In Proceedings of the IEEE, vol. 89, no. 1, January 2001.

[9] C.Cicconetti, A.Erta, L.Lenzini and E.Mingozzi, Performance Evaluation of the IEEE 802.16 MAC for QoS Support. In IEEE Transactions on Mobile Computing, vol. 6, no. 1, pp.26-38, January 2007.

[10] Tien V. Do, G. Buchholcz, D. D. Luong. Scheduling for broad-band wireless access with adaptive burst profiles. In The First International Wireless Summit (IWS 2005), (Denmark), September 2005.

[11] H.S. Wang and N. Moayeri. Modeling, Capacity, and Joint Source/Channel Coding for Rayleigh Fading Channels. In Proc. of $43^{\text {rd }}$ IEEE Vehicular Technology Conference, pp. 473479, 1993.

[12] Lingjie Li, Octavian Sarca FEC Performance with ARQ and Adaptive Burst Profile Selection. IEEE 802.16 working document, 2001.

\footnotetext{
${ }^{1}$ The CAPANINA project (http://www.capanina.org) involves
} 14 partners and is partially funded by the European Union.
[13] R. Jain and D. Chiu and W. Haweu A Quantitative Measure of Fairness and Dicrimination for Resource Allocation in Shared Computer System. Research Report, TR-301, September 1984.

[14] Ram Chakka and Tien V. Do, The MM $\backslash$ sum_ $\{\mathrm{k}=1\}^{\wedge} \mathrm{K}$ CPP_k/GE/c/L G-Queue with Heterogeneous Servers: Steady State Solution and an Application to Performance Evaluation, Performance Evaluation, Volume 64 , Issue 3 (March 2007), Pages: 191-209, ISSN:0166-5316.

\section{AUTHORS’ BIOGRAPHY}

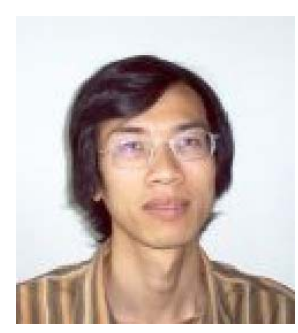

Nam Hoai Do received the M.Sc. in telecommunications engineering from the Technical University of Budapest, Hungary, in June 2006. He is currently a $\mathrm{PhD}$ student at the same university. His research interests include quality of service in wireless networks, the performance evaluation and planning of cross layered wireless systems, and scheduling algorithms for wireless networks.

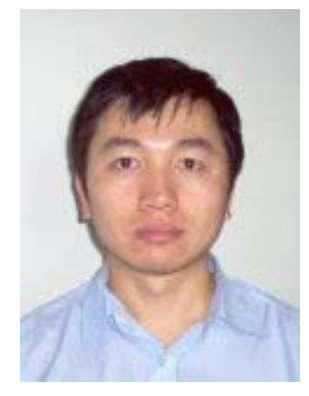

Dinh-Dung Luong received his Ms.C. and Ph.D. degrees from Budapest University of Technology and Economics (BUTE), Hungary, in 1998 and 2005, respectively. From 2005 to 2007, he was with the Multimedia Networks Laboratory at BUTE. Currently, he is a postdoctoral fellow with Management Networks and Telecommunications Research Laboratory at ETS, University of Quebec, where he conducts research and development in cognitive wireless mesh networks. His research interests also include network measurements, network management, congestion control, routing, MAC for wire- and wireless networks. 